\title{
A Novel Stability Analysis of Uncertain Switched Systems with Time-Varying Delays
}

\author{
Ganji Huang, ${ }^{1,2,3}$ Shixian Luo, ${ }^{2}$ Linna Wei, ${ }^{1,2,3}$ and Wuhua Chen ${ }^{2}$ \\ ${ }^{1}$ School of Mathematics, South China University of Technology, Guangzhou, Guangdong 510641, China \\ ${ }^{2}$ College of Mathematics and Information Science, Guangxi University, Nanning, Guangxi 530004, China \\ ${ }^{3}$ Guangxi Colleges and Universities Key Laboratory of Mathematics and Its Applications, Guangxi, China
}

Correspondence should be addressed to Ganji Huang; ganjih@163.com

Received 1 May 2015; Accepted 28 May 2015

Academic Editor: Zoltan Szabo

Copyright (C) 2015 Ganji Huang et al. This is an open access article distributed under the Creative Commons Attribution License, which permits unrestricted use, distribution, and reproduction in any medium, provided the original work is properly cited.

\begin{abstract}
This paper deals with the stability of switched systems with time-varying delay. The time-varying system parameters are assumed to be norm-bounded. Based on a novel switched time-varying Lyapunov functional method, some new LMI-based sufficient conditions have been obtained to ensure the exponential stability for the uncertain switched delays systems. Finally, the proposed method is applied to a numerical example and the simulative results are also given.
\end{abstract}

\section{Introduction}

Switched systems are essentially a kind of hybrid systems, which are made up of several subsystems and a switching rule. There are many reality systems that can be viewed as switched systems, for instance, robotic manufacture systems [1], power systems [2], transportation systems [3], and network control [4]. In addition, switched control has shown its advantages in stabilization of many nonlinear systems compared to using static feedback control strategy. Thus the problem of stability and control design of switched systems is of theoretical and practical importance.

As the foremost issue in the research of switched systems, stability analysis has gained a lot of attention and many important results have also been obtained during the several decades; see [5-9] and the references therein. Lyapunov function method plays a key role in stability analysis for switched systems. In general, there are two main methods: one is called common Lyapunov function (CLF) approach. The aim of this method is to construct a common Lyapunov function, which can ensure that the systems are stable under all possible switching signals [10-13]. Another more general technique is the so-called multiple Lyapunov function (MLF) method [14]. Different from the CLF approach, MLF method is to find one or more Lyapunov functions for each subsystem and combine them together to constitute a novel Lyapunov function. The research results have shown that MLF method can obtain less conservative results than by using CLF method [15-18].

As is well known, many reality systems' instability and poor behaviors are due in large to time delays. Switched delay systems are referred to as a class of switched systems with time delays in each subsystem, which has extensive application in engineering, such as networked control systems $[19,20]$ and power systems [21]. Because of their importance and widespread occurrence, stability analysis for switched systems with time delays has been a very active field in the last two decades [22-25]. It is worth noticing that there are few stability results reporting the uncertain switched system with time-varying delays. In [26], delay-dependent stability conditions and $L_{2}$-gain are obtained for switched systems by using average dwell time method combining LMI techniques. In [27], by using Lyapunov-Razumikhin functions method, stability conditions involving minimum dwell time are obtained for a class of switched time delays systems. Different from [26, 27], some delay-independent LMIbased sufficient conditions, which guarantee the stability of uncertain switched systems with state delays, are derived by constructing two novel Lyapunov functions/functionals in [28]. Exponential stability of a class of switched delays 
systems with state jumps is investigated in [29]; two stability results are obtained based on dwell time method. Recently, by constructing a new Lyapunov-Krasovskii functional and combining with average dwell time approach, [30] investigates the stability of a class of switched nonlinear systems with disturbance and delays; some conditions are proposed to ensure the input-to-state stability for the system.

In addition, note that stability of switched systems relates to the switching sequence besides subsystem [5]. This leads us to believe that an appropriate Lyapunov function/functional, which relates to the switching sequence, will be able to obtain less conservative results. However, CLF method or time-invariant MLF method is mainly used in the aforesaid results, and both methods often cannot make use of the information from the switching time. Thus, the convergence conditions based on CLF or time-invariant MLF approach may be conservative [31].

Inspired by the previous discussion, we will address the problem of stability for uncertain switched delays systems in this paper. We first develop a new type of piecewise time-varying Lyapunov functional and then use the novel Lyapunov functional combining Lyapunov-Krasovskii techniques to derive the exponential stability conditions for the switched systems. It will be shown that the new approach can get better results than the exiting results, and these stability conditions can be expressed in the form of linear matrix inequalities. Finally, an example will be given to show the effectiveness of the proposed approach.

\section{Problem Formulation}

In what follows, for matrix $A$, we use the notation $A>0$ $(\geq,<, \leq)$ to denote a symmetric positive-definite (positivesemidefinite, negative, and negative-semidefinite) matrix, and if not otherwise specified, all matrices are assumed to have suitable dimensions. The symbol $\|\cdot\|$ denotes the Euclidean norm for a vector. The symbol $I$ denotes an identity matrix, and $\mathbb{N}$ is a set of positive integers.

Consider the following switched system with uncertain and time delays:

$$
\begin{aligned}
\dot{x}(t) & =A_{\sigma(t)}(t) x(t)+B_{\sigma(t)}(t) x\left(t-\tau_{\sigma(t)}(t)\right), \\
x_{t_{0}}(\theta) & =\varphi(\theta), \quad \theta \in[-\tau, 0],
\end{aligned}
$$

where $x(t) \in \mathbb{R}^{n}$ is the system state vector, $\sigma(t):[0, \infty) \rightarrow$ $\mathscr{F} \triangleq\{1,2, \ldots, N\}$ is the switched signal function, and we define $\sigma(t)=k_{j} \in \mathscr{F}$ for $t \in\left[t_{j}, t_{j+1}\right)$, where $\left\{t_{j}\right\}$ is the $j$ th switching time instant. The time-varying matrices $A_{i}(t), B_{i}(t)$ express parameter uncertainties and satisfy

$$
\left[\begin{array}{ll}
A_{i}(t) & B_{i}(t)
\end{array}\right]=\left[\begin{array}{ll}
A_{i} & B_{i}
\end{array}\right]+D_{i} F_{i}(t)\left[\begin{array}{ll}
E_{i} & \bar{E}_{i}
\end{array}\right], \quad i \in \mathscr{F},
$$

where $A_{i}, B_{i}, D_{i}, E_{i}$, and $\bar{E}_{i}$ are known constant matrices which characterize the structure of the $i$ th subsystem and unknown time-varying matrices $F_{i}(t)$ satisfy $\left|F_{i}(t)\right| \leq 1$. $x_{t} \in P C([-\tau, 0], \mathbb{R})$ is defined by $x_{t}(\theta)=x(t+\theta)$ for $\theta \in[-\tau, 0]$ and $\varphi \in P C([-\tau, 0], \mathbb{R})$ is the initial function. $\tau_{i}(t), i \in \mathscr{F}$, denote the continuous time-varying delays of the $i$ th subsystem. In this paper, the time-varying delays $\tau_{i}(t)$ are assumed to satisfy the following condition:

$$
0 \leq \tau_{i}(t)=\tau(t) \leq \tau, \dot{\tau}(t) \leq d<1, \quad i \in \mathscr{F} .
$$

As mentioned before, stability performance of the switched system often depends on the switched signal. In this paper, we will consider the following two types of switching sequence:

$$
\begin{aligned}
& S\left(\delta_{1}, \delta_{2}\right)=\left\{\left\{t_{j}\right\} ; \delta_{1} \leq t_{j}-t_{j-1} \leq \delta_{2}, j \in \mathbb{N}\right\}, \\
& S\left(\delta_{1}, \infty\right)=\left\{\left\{t_{j}\right\} ; \delta_{1} \leq t_{j}-t_{j-1}, j \in \mathbb{N}\right\},
\end{aligned}
$$

where constants $\delta_{1}$ and $\delta_{2}$ satisfy $0<\delta_{1} \leq \delta_{2}$.

Definition 1. If, for arbitrary switching signal $\sigma(t) \in$ $S\left(\delta_{1}, \delta_{2}\right)$ (or $S\left(\delta_{1}, \infty\right)$ ) and any uncertainties satisfying (2), there exist constants $c, \lambda>0$ such that

$$
\|x(t)\| \leq c\|\varphi\| e^{-\lambda\left(t-t_{0}\right)}, \quad \forall t \geq t_{0},
$$

then system (1) is said to be robustly uniformly exponentially stable (RUES) over $S\left(\delta_{1}, \delta_{2}\right)$ (or $\left.S\left(\delta_{1}, \infty\right)\right)$.

Lemma 2 (see [32]). For any vectors $x, y \in \mathbb{R}^{n}$, matrices $A, P \in \mathbb{R}^{n \times n}, D \in \mathbb{R}^{n \times n_{f}}, E, N \in \mathbb{R}^{n_{f} \times n}$, and $F \in \mathbb{R}^{n_{f} \times n_{f}}$, with $P>0,\|F\| \leq 1$, and scalar $\varepsilon>0$, the following inequalities hold:

(i) $D F N+N^{T} F^{T} D^{T} \leq \varepsilon^{-1} D D^{T}+\varepsilon N^{T} N$.

(ii) $2 x^{T} y \leq x^{T} P^{-1} x+y^{T} P y$.

(iii) If $P-\varepsilon D D^{T}>0$, then

$$
\begin{gathered}
(A+D F(t) E)^{T} P^{-1}(A+D F(t) E) \\
\leq A^{T}\left(P-\varepsilon D D^{T}\right)^{-1} A+\varepsilon^{-1} E^{T} E .
\end{gathered}
$$

The main purpose of this paper is to develop new Lyapunov functional methods to analyze the stability of switched system (1) and to derive some conditions which can ensure switched system (1) is RUES over $S\left(\delta_{1}, \delta_{2}\right)$ (or $\left.S\left(\delta_{1}, \infty\right)\right)$.

\section{Main Results}

In this section, we first introduce several piecewise differentiable switched time-related functions, followed by a definition of a novel Lyapunov functional which will be applied to establish stability conditions for switched system (1). For given switching sequence $\left\{t_{j}\right\} \in S\left(\delta_{1}, \delta_{2}\right)$, we defined two time-varying functions $\rho, \rho_{1}:\left[t_{0}, \infty\right) \rightarrow \mathbb{R}^{+}$as follows:

$$
\begin{array}{r}
\rho(t)=\frac{t-t_{j}}{t_{j+1}-t_{j}}, \\
\rho_{1}(t)=\frac{1}{t_{j+1}-t_{j}}, \\
j \in \mathbb{N},
\end{array}
$$


for $t \in\left[t_{j}, t_{j+1}\right)$. It is easy to know

$$
\begin{gathered}
\rho(t) \in[0,1], \\
\rho\left(t_{j}\right)=0, \\
\rho\left(t_{j}^{-}\right)=1 .
\end{gathered}
$$

And there exists function $\rho_{2}(t) \in[0,1]$ satisfying

$$
\rho_{1}(t)=\frac{1-\rho_{2}(t)}{\delta_{1}}+\frac{\rho_{2}(t)}{\delta_{2}} .
$$

Furthermore, based on $\rho(t)$, we introduce a piecewise timevarying function as follows:

$$
\begin{aligned}
& \rho_{3}(t)= \begin{cases}\rho(t-\tau(t)), & t-\tau_{\sigma(t)}(t) \geq t_{0}, \\
1, & t-\tau_{\sigma(t)}(t) \in\left[t_{0}-\tau, t_{0}\right],\end{cases} \\
& \phi(t)=\left(\mu_{\sigma(t)}\right)^{\rho(t)-1},
\end{aligned}
$$

where scalars $\mu_{i} \geq 1, i \in \mathscr{F}$; then

$$
\begin{aligned}
& \rho_{3}(t) \in[0,1], \\
& \phi(t) \in\left[\left(\mu_{\sigma(t)}\right)^{-1}, 1\right], \quad t \geq t_{0} .
\end{aligned}
$$

Now we can introduce the following time-varying Lyapunov functional associated with $\rho(t)$ and $\phi(t)$ :

$$
\begin{aligned}
V\left(t, x_{t}\right)= & \phi(t) x^{\mathrm{T}}(t) P_{\sigma(t)}(t) x(t) \\
& +\int_{t-\tau(t)}^{t} \mathrm{e}^{-2 \gamma(t-s)} x^{\mathrm{T}}(s) Q(s) x(s) d s,
\end{aligned}
$$

where $P_{\sigma(t)}(t)=\rho(t) P_{\sigma(t), 1}+\tilde{\rho}(t) P_{\sigma(t), 2}, Q(t)=\rho(t) Q_{1}+\widetilde{\rho}(t) Q_{2}$, $\widetilde{\rho}(t)=1-\rho(t), P_{i, l}>0, Q_{l}>0, i \in \mathscr{F}, l=1,2$.

By applying time-varying Lyapunov functional (12) to system (1), we can obtain the following stability result.

Theorem 3. Consider switched system (1) with $\tau(t)$ satisfying condition (3) and $\left\{t_{j}\right\} \in S\left(\delta_{1}, \delta_{2}\right)$. If, for given scalars $\mu_{i} \geq 1$, $\gamma>0, i \in \mathscr{F}$, there exist matrices $P_{i, j}>0, Q_{j}>0, j=1,2$, and scalars $\varepsilon_{i j}>0, j=1,2$, such that the following matrix inequalities hold,

$$
\begin{aligned}
\left(\frac{1}{\mu_{i_{1}}}\right) P_{i_{1}, 2} & \leq P_{i_{2}, 1}, \quad \forall i_{1}, i_{2} \in \mathscr{F}, \\
\Theta_{i j l q} & =\left[\begin{array}{cccc}
\bar{\Omega}_{i j l} & P_{i j} B_{i} & P_{i j} D_{i} & P_{i j} D_{i} \\
* & \bar{\Phi}_{i q} & 0 & 0 \\
* & 0 & -\varepsilon_{i 1} I & 0 \\
* & 0 & 0 & -\varepsilon_{i 2} I
\end{array}\right]<0,
\end{aligned}
$$

for $i \in \mathscr{F}, j, l, q=1,2$, where

$$
\begin{aligned}
\bar{\Omega}_{i, j, l}= & \left(\frac{\ln \mu_{i}}{\delta_{1}}+2 \gamma\right) P_{i, j}+P_{i, j} A_{i}+A_{i}^{T} P_{i, j} \\
& +\frac{1}{\delta_{l}}\left(P_{i, 1}-P_{i, 2}\right)+\varepsilon_{i 1} E_{i}^{T} E_{i}+\mu_{i} Q_{j}, \\
\bar{\Phi}_{i, q}= & \varepsilon_{i 2} \bar{E}_{i}^{T} \bar{E}_{i}-(1-d) \exp (-2 \gamma \tau) Q_{q},
\end{aligned}
$$

then switched system (1) is RUES over $S\left(\delta_{1}, \delta_{2}\right)$.
Proof. Let $\lambda_{1}=\min \left\{\lambda_{\min }\left(P_{i, j}\right) ; i \in \mathscr{F}, j=1,2\right\}, \lambda_{2}=$ $\max \left\{\lambda_{\max }\left(P_{i, j}\right) ; i \in \mathscr{F}, \dot{j}=1,2\right\}, \lambda_{3}=\max \left\{\lambda_{\text {max }}\left(Q_{j}\right) ; j=\right.$ $1,2\}$, and $\mu=\max \left\{\left(\mu_{i}\right) ; i \in \mathscr{F}\right\}$. Thus, by the definition of $V(t, x(t)),(8)$, and (11), we have

$$
\frac{1}{\mu} \lambda_{1}\|x(t)\|^{2} \leq V(t, x(t)) \leq\left(\lambda_{2}+\lambda_{3}\right)\|x(t)\|^{2} .
$$

Set $V(t)=V(t, x(t))$, for $t \in\left[t_{j}, t_{j+1}\right)$, and, applying Lemma 2, the upper right-hand derivative of $V(t)$ along the trajectories of system (1) is given by

$$
\begin{aligned}
& D^{+} V(t) \leq \phi(t)\left(x ^ { \mathrm { T } } ( t ) \left(\frac{\ln \left(\mu_{k_{j}}\right)}{\delta_{1}} P_{k_{j}}(t)\right.\right. \\
& +\rho_{1}(t)\left(P_{k_{j}, 1}-P_{k_{j}, 2}\right)+2 P_{k_{j}}(t) A_{k_{j}} \\
& \left.+\left(\varepsilon_{k_{j} 1}^{-1}+\varepsilon_{k_{j} 2}^{-1}\right) P_{k_{j}}(t) D_{k_{j}} D_{k_{j}}^{\mathrm{T}} P_{k_{j}}(t)+\varepsilon_{k_{j} 1} E_{k_{j}}^{\mathrm{T}} E_{k_{j}}\right) \\
& \cdot x(t)+2 x^{\mathrm{T}}(t) P_{k_{j}}(t) B_{k_{j}} x\left(t-\tau_{k_{j}}(t)\right) \\
& +2 x^{\mathrm{T}}(t) P_{k_{j}}(t) B_{k_{j}} x\left(t-\tau_{k_{j}}(t)\right)+\varepsilon_{k_{j}} x^{\mathrm{T}}(t \\
& \left.\left.-\tau_{k_{j}}(t)\right) \bar{E}_{k_{j}}^{\mathrm{T}} \bar{E}_{k_{j}} x\left(t-\tau_{k_{j}}(t)\right)\right)+x^{\mathrm{T}}(t) Q(t) x(t) \\
& -2 \gamma \int_{t-\tau_{k_{j}}(t)}^{t} \mathrm{e}^{-2 \gamma(t-s)} x^{\mathrm{T}}(s) \mathrm{Q}(t) x(s) d s-(1-d) \\
& \cdot \mathrm{e}^{-2 \gamma \tau} x^{\mathrm{T}}\left(t-\tau_{k_{j}}(t)\right) Q\left(t-\tau_{k_{j}}(t)\right) x\left(t-\tau_{k_{j}}(t)\right) \\
& \leq-2 \gamma V_{2}(t)+\phi(t)\left(x^{\mathrm{T}}(t)\right. \\
& \left(\left(\frac{\ln \left(\mu_{k_{j}}\right)}{\delta_{1}}+2 \gamma\right) P_{k_{j}}(t)+\rho_{1}(t)\left(P_{k_{j}, 1}-P_{k_{j}, 2}\right)\right. \\
& +2 P_{k_{j}}(t) A_{k_{j}}+\left(\varepsilon_{k_{j} 1}^{-1}+\varepsilon_{k_{j} 2}^{-1}\right) P_{k_{j}}(t) D_{k_{j}} D_{k_{j}}^{\mathrm{T}} P_{k_{j}}(t) \\
& \left.+\varepsilon_{k_{j} 1} E_{k_{j}}^{\mathrm{T}} E_{k_{j}}+\mu_{k_{j}} Q(t)\right) x(t)+2 x^{\mathrm{T}}(t) P_{k_{j}}(t) \\
& \cdot B_{k_{j}} x\left(t-\tau_{k_{j}}(t)\right)+\varepsilon_{k_{j} 2} x^{\mathrm{T}}\left(t-\tau_{k_{j}}(t)\right) \\
& \left.\cdot \bar{E}_{k_{j}}^{\mathrm{T}} \bar{E}_{k_{j}} x\left(t-\tau_{k_{j}}(t)\right)\right)-(1-d) \mathrm{e}^{-2 \gamma(t-s)} x^{\mathrm{T}}(t \\
& \left.-\tau_{k_{j}}(t)\right) Q\left(t-\tau_{k_{j}}(t)\right) x\left(t-\tau_{k_{j}}(t)\right)=-2 \gamma V(t) \\
& +\phi(t) \eta^{\mathrm{T}}(t) \bar{\Theta}_{k_{j}} \eta(t)
\end{aligned}
$$


where $\eta^{\mathrm{T}}(t)=\left[\begin{array}{ll}x^{\mathrm{T}}(t) & x^{\mathrm{T}}\left(t-\tau_{k_{j}}(t)\right)\end{array}\right]$ and

$$
\begin{aligned}
\bar{\Theta}_{k_{j}}= & {\left[\begin{array}{cc}
\bar{\Omega}_{k_{j}} & P_{k_{j}}(t) B_{k_{j}} \\
* & \bar{\Phi}_{k_{j}}
\end{array}\right], } \\
\bar{\Omega}_{k_{j}}= & \left(\frac{\ln \left(\mu_{k_{j}}\right)}{\delta_{1}}+2 \gamma\right) P_{k_{j}}(t)+\rho_{1}(t)\left(P_{k_{j} 1}-P_{k_{j} 2}\right) \\
& +2 P_{k_{j}}(t) A_{k_{j}} \\
& +\left(\varepsilon_{k_{j} 1}^{-1}+\varepsilon_{k_{j} 2}^{-1}\right) P_{k_{j}}(t) D_{k_{j}} D_{k_{j}}^{\mathrm{T}} P_{k_{j}}(t) \\
& +\varepsilon_{k_{j} 1} E_{k_{j}}^{\mathrm{T}} E_{k_{j}}+\mu_{k_{j}} Q(t), \\
\bar{\Phi}_{k_{j}}= & \varepsilon_{k_{j} 2} \bar{E}_{k_{j}}^{\mathrm{T}} \bar{E}_{k_{j}}-(1-d) \exp (-2 \gamma \tau) Q\left(t-\tau_{k_{j}}(t)\right) .
\end{aligned}
$$

Set $\widetilde{\rho}_{2}(t)=1-\rho_{2}(t), \widetilde{\rho}_{3}(t)=1-\rho_{3}(t)$, and, using (9) and (14), it is easy to see that

$$
\begin{aligned}
\rho(t) & {\left[\rho_{2}(t)\left(\rho_{3}(t) \Theta_{k_{0} 121}+\tilde{\rho}_{3}(t) \Theta_{k_{0} 122}\right)\right.} \\
+ & \left.\widetilde{\rho}_{2}(t)\left(\rho_{3} \Theta_{k_{0} 111}+\widetilde{\rho}_{3}(t) \Theta_{k_{0} 112}\right)\right]+\widetilde{\rho}(t) \\
\cdot & {\left[\rho_{2}(t)\left(\rho_{3}(t) \Theta_{k_{0} 221}+\widetilde{\rho}_{3}(t) \Theta_{k_{0} 222}\right)\right.} \\
+ & \left.\tilde{\rho}_{2}(t)\left(\rho_{3} \Theta_{k_{0} 211}+\tilde{\rho}_{3}(t) \Theta_{k_{0} 212}\right)\right]<0 .
\end{aligned}
$$

The above inequality can be rewritten as the following form:

$$
\left[\begin{array}{cccc}
\widetilde{\Omega}_{k_{j}} & P_{k_{j}}(t) B_{k_{j}} & P_{k_{j}}(t) D_{k_{j}} & P_{k_{j}}(t) D_{k_{j}} \\
* & \widetilde{\Phi}_{k_{j}} & 0 & 0 \\
* & 0 & -\varepsilon_{k_{j} 1} I & 0 \\
* & 0 & 0 & -\varepsilon_{k_{j} 2} I
\end{array}\right]<0
$$

where

$$
\begin{aligned}
\widetilde{\Omega}_{k_{j}}= & \left(\frac{\ln \mu_{i}}{\delta_{1}}+2 \gamma\right) P_{k_{j}}(t)+P_{k_{j}}(t) A_{k_{j}}+A_{k_{j}}^{\mathrm{T}} P_{k_{j}}(t) \\
& +\rho_{1}(t)\left(P_{k_{j}, 1}-P_{k_{j}, 2}\right)+\varepsilon_{k_{j} 1} E_{k_{j}}^{\mathrm{T}} E_{k_{j}} \\
& +\mu_{k_{j}} Q(t), \\
\widetilde{\Phi}_{k_{j}}= & \varepsilon_{k_{j} 2} \bar{E}_{k_{j}}^{\mathrm{T}} \bar{E}_{k_{j}}-(1-d) \exp (-2 \gamma \tau) Q\left(t-\tau_{k_{j}}(t)\right) .
\end{aligned}
$$

Using Schur complement, we can obtain $\bar{\Theta}_{k_{j}}<0$. It follows from (17) that, for $t \in\left[t_{j}, t_{j+1}\right)$, we have $D^{+} V(t) \leq-2 \gamma V(t)$, which implies

$$
V(t) \leq V\left(t_{j}^{+}\right) \exp \left(-2 \gamma\left(t-t_{j}\right)\right)
$$

On the other hand, at switching time $t_{j}$, applying (8), (11), and (13), we obtain

$$
\begin{aligned}
V\left(t_{j}\right) & \\
= & \phi\left(t_{j}\right) x^{\mathrm{T}}\left(t_{j}\right)\left(\rho\left(t_{j}\right) P_{k_{j}, 1}+\tilde{\rho}\left(t_{j}\right) P_{k_{j}, 2}\right) x\left(t_{j}\right) \\
& +\int_{t_{j}-\tau\left(t_{j}\right)}^{t_{j}} \mathrm{e}^{-2 \gamma(t-s)} x^{\mathrm{T}}(s) Q(s) x(s) d s \\
= & \frac{1}{\mu_{k_{j}}} x^{\mathrm{T}}\left(t_{j}^{-}\right) P_{k_{j}, 2} x\left(t_{j}^{-}\right) \\
& +\int_{t_{j}-\tau\left(t_{j}\right)}^{t_{j}} \mathrm{e}^{-2 \gamma(t-s)} x^{\mathrm{T}}(s) Q(s) x(s) d s \\
\leq & x^{\mathrm{T}}\left(t_{j}^{-}\right) P_{k_{j}-1,1} x\left(t_{j}^{-}\right) \\
& +\int_{t_{j}-\tau\left(t_{j}\right)}^{t_{j}} \mathrm{e}^{-2 \gamma(t-s)} x^{\mathrm{T}}(s) Q(s) x(s) d s=V\left(t_{j}^{-}\right) .
\end{aligned}
$$

For any given $t \geq t_{0}$, there exists $j_{0} \in \mathbb{N}$, such that $t \in$ $\left[t_{j_{0}}, t_{j_{0}+1}\right)$. Combining (22) and (23) together yields

$$
\begin{aligned}
V(t) & \leq V\left(t_{j_{0}}\right) \exp \left(-2 \gamma\left(t-t_{j_{0}}\right)\right) \\
& \leq V\left(t_{j_{0}}^{-}\right) \exp \left(-2 \gamma\left(t-t_{j_{0}}\right)\right) \\
& \leq V\left(t_{j_{0}-1}\right) \exp \left(-2 \gamma\left(t-t_{j_{0}-1}\right)\right) \leq \cdots \\
& \leq V\left(t_{0}\right) \exp \left(-2 \gamma\left(t-t_{0}\right)\right) .
\end{aligned}
$$

So by this inequality and (16), we obtain

$$
\begin{aligned}
& \|x(t)\| \leq \sqrt{\frac{\mu\left(\lambda_{2}+\lambda_{3}\right)}{\lambda_{1}}}\|\varphi\| \exp \left(-\gamma\left(t-t_{0}\right)\right), \\
& t>t_{0} \text {, }
\end{aligned}
$$

which implies that switched system (1) with $\tau(t)$ satisfying condition (3) is RUES over $S\left(\delta_{1}, \delta_{2}\right)$.

Remark 4. The stability analysis of system (1) is performed by applying the Lyapunov functional $V(t)$ given in (12). In $V(t)$, the time-varying matrices $P_{\sigma(t)}(t)$ and $Q(t)$ are time-varying convex combinations of two constant positive matrices, in which the combination are related to the switched time sequences. However, the traditional Lyapunov functional is usually chosen the case of $V(t)$ when $P_{\sigma(t), 1}=P_{\sigma(t), 2}$ (i.e., $\left.P_{i 1}=P_{i 2}, i \in \mathscr{F}\right)$ and $Q_{1}=Q_{2}$. Therefore, compared with the traditional Lyapunov functional, the time-varying Lyapunov functional can capture the switched characteristic of the considered system and bring less conservative results.

In the case of $\left\{t_{j}\right\} \in S\left(\delta_{1}, \infty\right)$, based on Theorem 3, we have the following corollary.

Corollary 5. Consider switched system (1) with $\tau(t)$ satisfying condition (3) and $\left\{t_{j}\right\} \in S\left(\delta_{1}, \infty\right)$. If, for given scalars $\mu_{i} \geq 1$, 
$\gamma>0, i \in \mathscr{F}$, there exist matrices $P_{i}>0, Q_{j}>0, j=1,2$, and scalars $\varepsilon_{i j}>0, j=1,2$, such that the following matrix inequalities hold,

$$
\begin{aligned}
\left(\frac{1}{\mu_{i_{1}}}\right) P_{i_{1}} \leq & P_{i_{2}}, \quad \forall i_{1}, i_{2} \in \mathscr{F}, \\
\Theta_{i j q} & =\left[\begin{array}{cccc}
\bar{\Omega}_{i j} & P_{i} B_{i} & P_{i} D_{i} & P_{i} D_{i} \\
* & \bar{\Phi}_{i q} & 0 & 0 \\
* & 0 & -\varepsilon_{i 1} I & 0 \\
* & 0 & 0 & -\varepsilon_{i 2} I
\end{array}\right]<0,
\end{aligned}
$$

for $i \in \mathscr{F}, j, q=1,2$, where

$$
\begin{aligned}
\bar{\Omega}_{i, j}= & \left(\frac{\ln \mu_{i}}{\delta_{1}}+2 \gamma\right) P_{i}+P_{i} A_{i}+A_{i}^{T} P_{i}+\varepsilon_{i 1} E_{i}^{T} E_{i} \\
& +\mu_{i} Q_{j}, \\
\bar{\Phi}_{i, q}= & \varepsilon_{i 2} \bar{E}_{i}^{T} \bar{E}_{i}-(1-d) \exp (-2 \gamma \tau) Q_{q},
\end{aligned}
$$

then switched system (1) with $\tau(t)$ satisfying condition (3) is RUES over $S\left(\delta_{1}, \infty\right)$.

Proof. Suppose that LMIs (26) are feasible. Choosing $P_{i, j}=P_{i}$, $i \in \mathscr{F}, j=1,2$, then one can verify that matrix inequalities (13) and (14) are also feasible.

Remark 6. In the earlier discussion, we have assumed that there are no impulsive effects at the switching points. However, similar approach can also be used in the stability analysis for impulsive switched systems.

Remark 7. We can also make a further study in the problem of stabilization for system (1) by using the method proposed in this paper. In the stabilization setting, different subsystem has different controller gain and Lyapunov matrix, so a key question is how to convert the stabilization issue to solve a set of LMIs; at this point, one can refer to the recent paper [31], where the problem of observers design for switched linear systems has been tackled.

\section{Numerical Examples}

In this section, we will give an example to show the effectiveness of our new approach.

Example 1. Consider switched system (1) with two subsystems, and the parameters are given as follows:

$$
\begin{aligned}
& A_{1}=\left[\begin{array}{cc}
0 & 1 \\
-10 & -1
\end{array}\right], \\
& A_{2}=\left[\begin{array}{cc}
0 & 1 \\
-0.1 & -0.5
\end{array}\right], \\
& B_{1}=\theta_{1}\left[\begin{array}{cc}
0.1 & 0 \\
-0.01 & 0.05
\end{array}\right],
\end{aligned}
$$

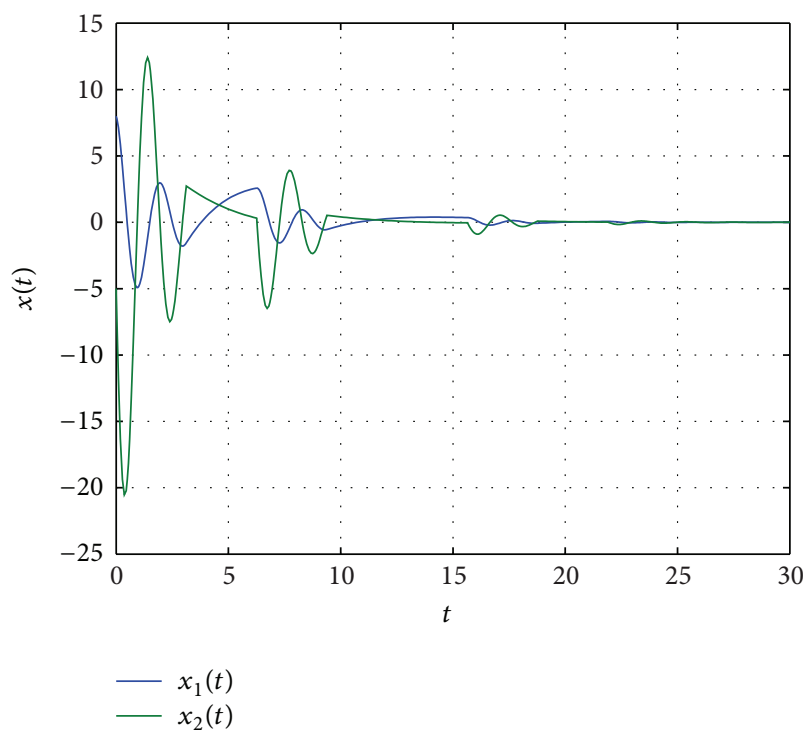

FIGURE 1: State responses of the system.

$$
\begin{aligned}
B_{2} & =\theta_{2}\left[\begin{array}{cc}
0.02 & 0 \\
-0.01 & 0.02
\end{array}\right], \\
\sigma(t) & \in\{1,2\} .
\end{aligned}
$$

In order to compare our results with the existing ones, we calculate the lower bound $\delta_{1}$ on the minimum dwell time from three cases as followings.

Case $1\left(\theta_{i}=0, E_{i}=\bar{E}_{i}=D_{i}=0, i=1,2\right)$. By applying Theorem 3 with choice of $\mu_{1}=21.6, \mu_{2}=1.01$, the lower bound of the minimum dwell time $\delta_{1}$ that can be found is 3.13 . In the results of $[27,28]$, it was found that the corresponding lower bound of the minimum dwell time is 3.42 and 34.8579 , respectively.

Case $2\left(\theta_{i}=1, E_{i}=\bar{E}_{i}=D_{i}=0, i=1,2\right)$. By applying Theorem 3 and choosing $\mu_{1}=4.5, \mu_{2}=1$, the obtained lower bound of the minimum dwell time is $\delta_{1}=12.18$. By Theorem 1 in [26], the average dwell time is 14.79. It was reported in [28] that the lower bound of the minimum dwell time is $\delta_{1}=14.19$.

Case $3\left(\theta_{i}=1, D_{i}=0.1 I, i=1,2, E_{1}=\bar{E}_{1}=0.02 I\right.$, $\left.E_{2}=\bar{E}_{2}=0.08 I\right)$. We note that, for this case, the condition of [28] cannot be satisfied. However, by using Theorem 3 in this paper and choosing $\mu_{1}=2.6$ and $\mu_{2}=1.025$, the obtained lower bound of the minimum dwell time is $\delta_{1}=22$.

Based on the analysis above, our result is much less conservative than the results of [26-28]. For simulation studies, we consider Case 1. Choose dwell time $\delta_{1}=3.14$. With the initial functions $x(0)=\left[\begin{array}{ll}8 & -5\end{array}\right]^{\mathrm{T}}$, the time response curves of the system are plotted in Figure 1 and the switching signal is shown in Figure 2. 


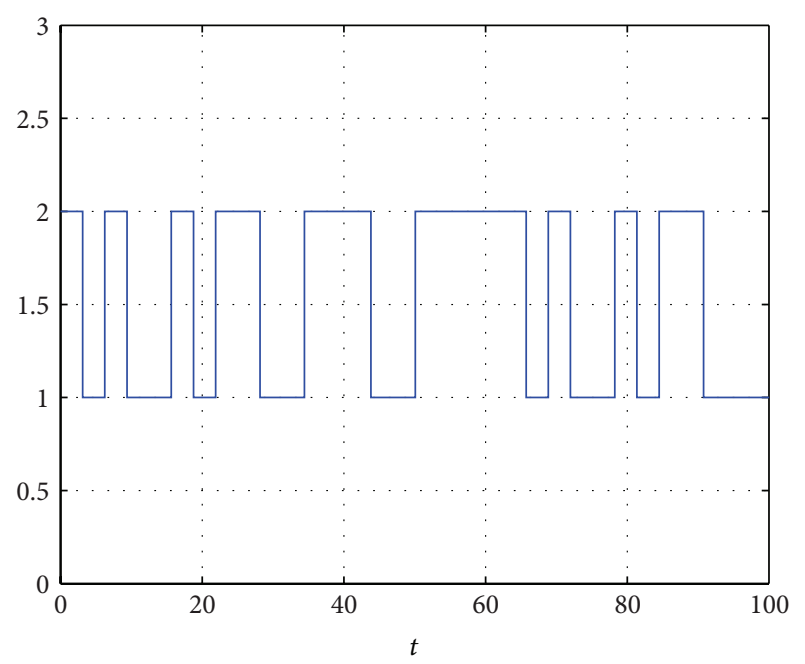

FIGURE 2: Switching signal $\sigma(t)$ with minimum dwell time $\delta_{1}=3.13$.

\section{Conclusion}

By introducing a new type of Lyapunov functional method, some delay-dependent sufficient conditions of stability for a class of switched systems with uncertain and state delays have been presented, and these conditions can be expressed in the form of LMI. The numerical analysis results have shown that the proposed approach can obtain better results than the earlier results.

\section{Conflict of Interests}

The authors declare that there is no conflict of interests related to this paper.

\section{Acknowledgments}

This work was supported by the National Natural Science Foundation of China under Grant no. 61164016, Guangxi Natural Science Foundations (2013GXNSFDA019003), Guangxi Colleges and Universities Key Laboratory of Mathematics and Its Applications, the Innovation Project of Guangxi Graduate Education (YCSZ2015031), and Scientific Research Foundation of Guangxi University (Grant no. XJZ110626).

\section{References}

[1] J. Tan, N. Xi, and Y. Wang, "A singularity-free motion control algorithm for robot manipulators-a hybrid system approach," Automatica, vol. 40, no. 7, pp. 1239-1245, 2004.

[2] Y. B. Shtessel, O. A. Raznopolov, and L. A. Ozerov, "Control of multiple modular DC-to-DC power converters in conventional and dynamic sliding surfaces," IEEE Transactions on Circuits and Systems I: Fundamental Theory and Applications, vol. 45, no. 10, pp. 1091-1100, 1998.

[3] R. Horowitz and P. Varaiya, "Control design of an automated highway system," Proceedings of the IEEE, vol. 88, no. 7, pp. 913$925,2000$.
[4] P. V. Zhivoglyadov and R. H. Middleton, "Networked control design for linear systems," Automatica, vol. 39, no. 4, pp. 743750, 2003.

[5] R. A. Decarlo, M. S. Branicky, S. Pettersson, and B. Lennartson, "Perspectives and results on the stability and stabilizability of hybrid systems," Proceedings of the IEEE, vol. 88, no. 7, pp. 1069$1082,2000$.

[6] D. Liberzon, Switching in Systems and Control, Birkhäuser, Boston, Mass, USA, 2003.

[7] D. Liberzon and A. S. Morse, "Basic problems in stability and design of switched systems," IEEE Control Systems Magazine, vol. 19, no. 5, pp. 59-70, 1999.

[8] Z. Sun and S. S. Ge, Switched Linear System: Control and Design, Springer, New York, NY, USA, 2005.

[9] H. Lin and P. J. Antsaklis, "Stability and stabilizability of switched linear systems: a survey of recent results," IEEE Transactions on Automatic Control, vol. 54, no. 2, pp. 308-322, 2009.

[10] R. N. Shorten and K. S. Narendra, "Necessary and sufficient conditions for the existence of a common quadratic Lyapunov function for a finite number of stable second order linear timeinvariant systems," International Journal of Adaptive Control and Signal Processing, vol. 16, no. 10, pp. 709-728, 2002.

[11] Y. G. Sun, L. Wang, and G. M. Xie, "Stability of switched systems with time-varying delays: delay-dependent common Lyapunov functional approach," in Proceedings of the American Control Conference, pp. 1544-1549, IEEE, Minneapolis, Minn, USA, June 2006.

[12] D. Liberzon and R. Tempo, "Common Lyapunov functions and gradient algorithms," IEEE Transactions on Automatic Control, vol. 49, no. 6, pp. 990-994, 2004.

[13] Y. G. Sun and L. Wang, "On stability of a class of switched nonlinear systems," Automatica, vol. 49, no. 1, pp. 305-307, 2013.

[14] M. S. Branicky, "Multiple Lyapunov functions and other analysis tools for switched and hybrid systems," IEEE Transactions on Automatic Control, vol. 43, no. 4, pp. 475-482, 1998.

[15] J. P. Hespanha, D. Liberzon, D. Angeli, and E. D. Sontag, "Nonlinear norm-observability notions and stability of switched systems," IEEE Transactions on Automatic Control, vol. 50, no. 2, pp. 154-168, 2005.

[16] J. Zhao and D. J. Hill, "On stability, $L_{2}$-gain and $H_{\infty}$ control for switched systems," Automatica, vol. 44, no. 5, pp. 1220-1232, 2008.

[17] R. Kuiava, R. A. Ramos, H. R. Pota, and L. F. Alberto, "Practical stability of switched systems without a common equilibria and governed by a time-dependent switching signal," European Journal of Control, vol. 19, no. 3, pp. 206-213, 2013.

[18] D. Xie, H. Zhang, and B. Wang, "Exponential stability of switched systems with unstable subsystems: a mode-dependent average dwell time approach," Circuits, Systems, and Signal Processing, vol. 32, no. 6, pp. 3093-3105, 2013.

[19] G.-P. Liu, Y. Xia, D. Rees, and W. S. Hu, "Design and stability criteria of networked predictive control systems with random network delay in the feedback channel," IEEE Transactions on Systems, Man and Cybernetics Part C: Applications and Reviews, vol. 37, no. 2, pp. 173-184, 2007.

[20] D. Xie, Y. Wu, and X. Chen, "Stabilization of discrete-time switched systems with input time delay and its applications in networked control systems," Circuits, Systems, and Signal Processing, vol. 28, no. 4, pp. 595-607, 2009. 
[21] F. F. Digham and M.-S. Alouini, "Variable-rate noncoherent M-FSK modulation for power limited systems over Nakagamifading channels," IEEE Transactions on Wireless Communications, vol. 3, no. 4, pp. 1295-1304, 2004.

[22] L. V. Hien, Q. P. Ha, and V. N. Phat, "Stability and stabilization of switched linear dynamic systems with time delay and uncertainties," Applied Mathematics and Computation, vol. 210, no. 1, pp. 223-231, 2009.

[23] X.-M. Sun, W. Wang, G.-P. Liu, and J. Zhao, "Stability analysis for linear switched systems with time-varying delay," IEEE Transactions on Systems, Man, and Cybernetics Part B: Cybernetics, vol. 38, no. 2, pp. 528-533, 2008.

[24] W. A. Zhang and L. Yu, "Stability analysis for discrete-time switched time-delay systems," Automatica, vol. 45, no. 10, pp. 2265-2271, 2009.

[25] Z. C. Li, H. J. Gao, and H. R. Karimi, "Stability analysis and $H_{\infty}$ controller synthesis of discrete-time switched systems with time delay," Systems and Control Letters, vol. 66, pp. 85-93, 2014.

[26] X.-M. Sun, J. Zhao, and D. J. Hill, "Stability and $L_{2}$-gain analysis for switched delay systems: a delay-dependent method," Automatica, vol. 42, no. 10, pp. 1769-1774, 2006.

[27] P. Yan and H. Özbay, "Stability analysis of switched time delay systems," SIAM Journal on Control and Optimization, vol. 47, no. 2, pp. 936-949, 2008.

[28] W.-H. Chen and W. X. Zheng, "Delay-independent minimum dwell time for exponential stability of uncertain switched delay systems," IEEE Transactions on Automatic Control, vol. 55, no. 10, pp. 2406-2413, 2010.

[29] C. D. Li, T. W. Huang, G. Feng, and G. R. Chen, "Exponential stability of time-controlled switching systems with time delay," Journal of the Franklin Institute, vol. 349, no. 1, pp. 216-233, 2012.

[30] Y.-E. Wang, X.-M. Sun, Z. Wang, and J. Zhao, "Construction of Lyapunov-Krasovskii functionals for switched nonlinear systems with input delay," Automatica, vol. 50, no. 4, pp. 12491253, 2014.

[31] G. J. Huang and W. H. Chen, "A revisit to the design of switched observers for switched linear systems with unknown inputs," International Journal of Control, Automation and Systems, vol. 12, no. 5, pp. 954-962, 2014.

[32] W.-H. Chen, J.-G. Wang, Y.-J. Tang, and X. Lu, "Robust $H_{\infty}$ control of uncertain linear impulsive stochastic systems," International Journal of Robust and Nonlinear Control, vol. 18, no. 13, pp. 1348-1371, 2008. 

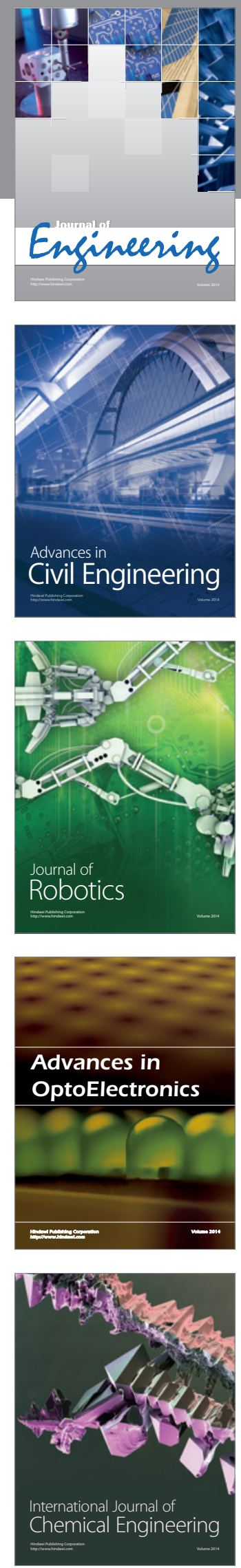

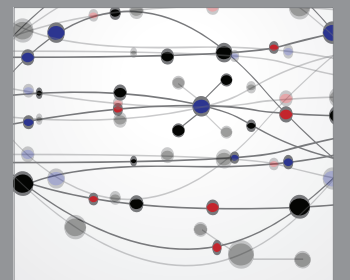

The Scientific World Journal
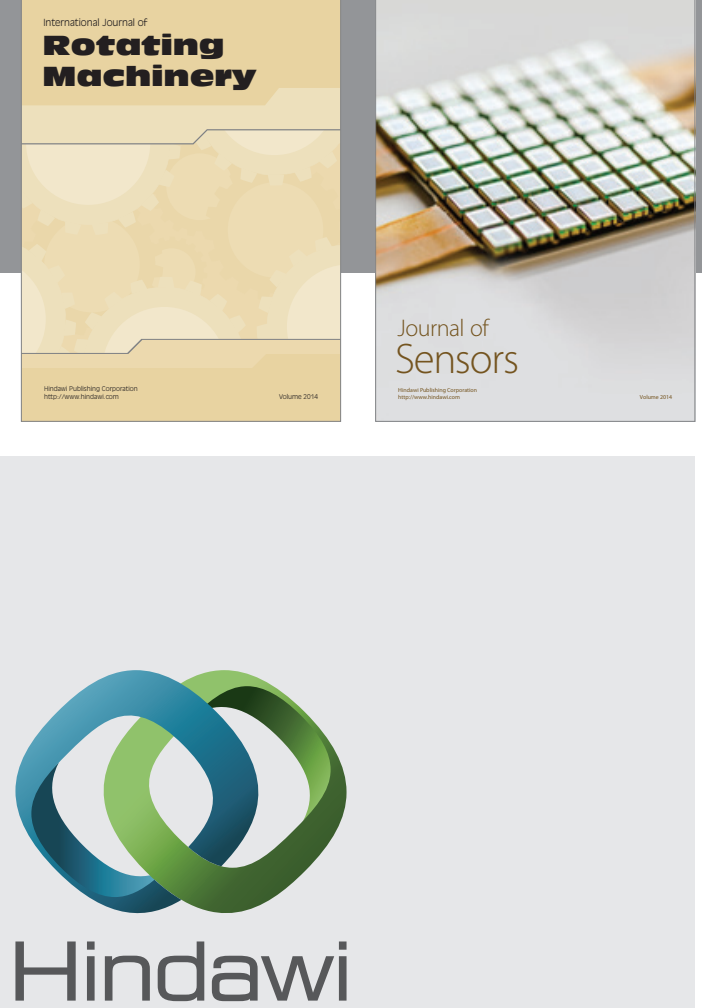

Submit your manuscripts at http://www.hindawi.com

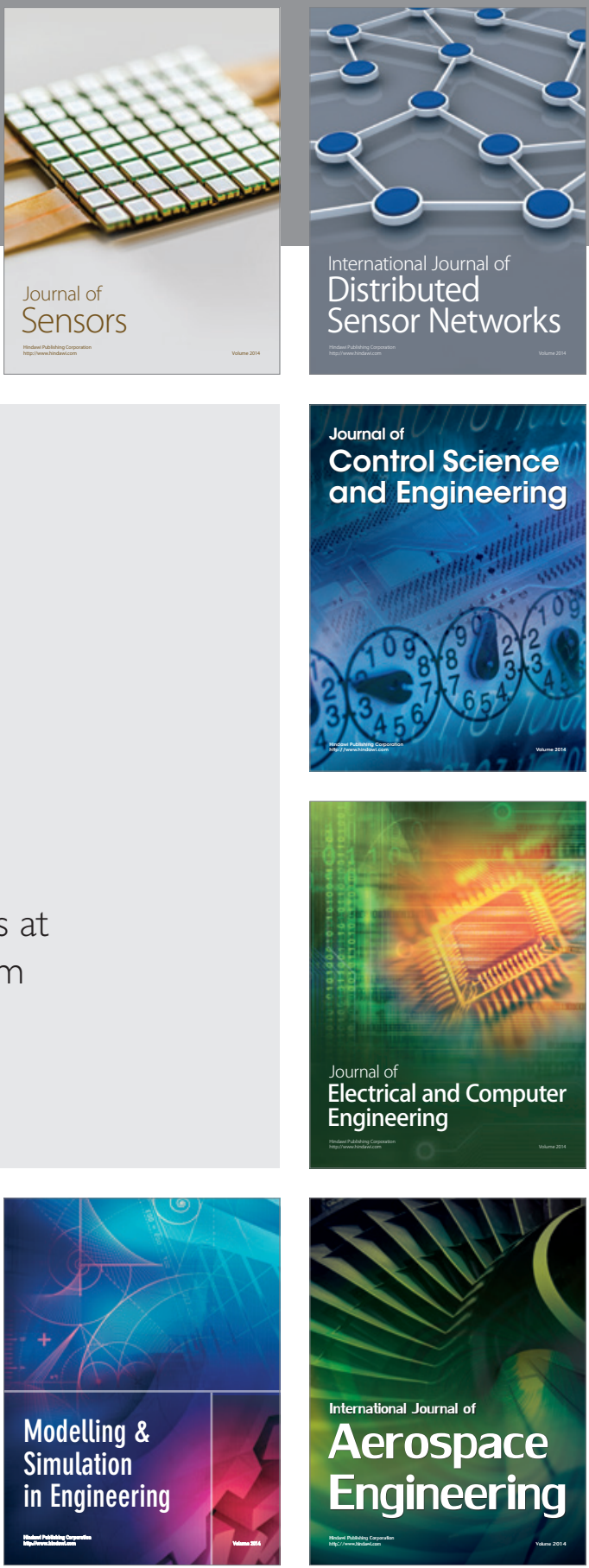

Journal of

Control Science

and Engineering
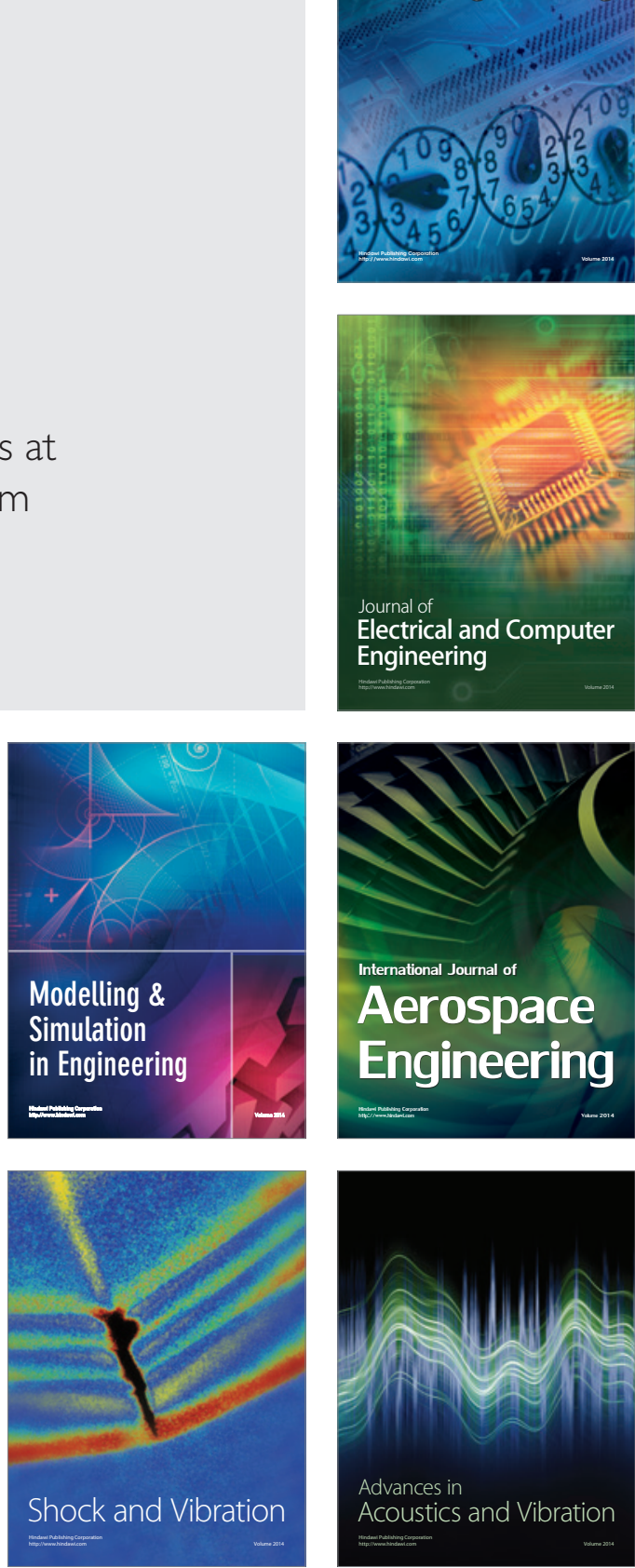